\title{
INFLUENCIA DEL RECUBRIMIENTO DE CROMATO DE ZINC EN LA CORROSIÓN DE LOS ACEROS ASTM A-500 Y A-500 GALVANIZADO EXPUESTOS EN UNA CÁMARA DE NIEBLA SALINA
}

\section{InfluenCE OF THE ZinC Chromate COATING ON THE CORROSION OF ASTM A-500 AND GALVANIZED A-500 STEEL EXPOSED INTO A SALT FOG CORROSION CHAMBER}

\author{
Cristian Guilcaso $^{1, *}$ (D), Leonidas Ramírez ${ }^{2}$ (D), Augusto Coque ${ }^{3}$ (D), Xavier Vaca² (D), \\ Diego Molina ${ }^{1}$ (D), Isaac Simbaña ${ }^{2}$ (iD
}

Recibido: 13-11-2020, Recibido tras revisión: 04-02-2021, Aceptado: 09-03-2021, Publicado: 01-07-2021

\section{Resumen}

En esta investigación se ha analizado la influencia del recubrimiento de cromato de zinc en la corrosión del acero ASTM A-500 y A-500 galvanizado expuesto en una cámara de niebla salina acorde a la norma ASTM B117. Se realizaron dos métodos de limpieza superficial antes de la aplicación del recubrimiento, según la normas SSPC-SP-3 y SSPC-SP-5, una limpieza mecánica y otra con chorro presurizado. Las probetas se introdujeron en la cámara con tiempos de exposición de 200, 250 y 350 h. Se utilizaron diferentes equipos para registrar información que fue utilizada en el cálculo de la velocidad de corrosión. Con evaluaciones visuales, utilizando las normas ASTM D-610 y ASTM D-714, se determinó el grado de corrosión de las probetas y la frecuencia de ampollas, respectivamente. Se comparó los materiales sin recubrimiento y los dos métodos de limpieza superficial. Los resultados obtenidos han demostrado que el acero galvanizado presentó una menor velocidad de corrosión.

Palabras clave: ASTM-500, recubrimientos anticorrosivos, cámara de niebla salina

\begin{abstract}
In this research work it has been analyzed the influence of the Zinc chromate coating on the corrosion of ASTM A-500 and galvanized A-500 steels exposed in a salt spray corrosion chamber, according to the ASTM B117 Standard. Two surface cleaning methods were used prior to applying the coating, considering the SSPC-SP-3 and SSP-SP-5 standards, namely a mechanical cleaning and a blast cleaning. The samples were put into the chamber with exposure times of 200, 250 and $350 \mathrm{~h}$. Different equipment were used for recording the information that was used to calculate the corrosion rate. Through visual assessments according to the ASTM-D610 and ASTM D-714 standards, the corrosion degree and the blistering frequency, respectively, were determined. The materials without coating and coated after the two surface cleaning methods were compared. The results obtained have demonstrated that galvanized steel exhibited a lower corrosion rate.
\end{abstract}

Keywords: ASTM-500, anticorrosive coatings, salt spray corrosion chamber

\footnotetext{
1,*Facultad de Ingeniería Civil y Mecánica, Universidad Técnica de Ambato, Ambato, Ecuador.

Autor para correspondencia cguilcaso6706@uta.edu.ec.

${ }^{2}$ Carrera de Ingeniería Mecánica, Universidad Politécnica Salesiana, Quito, Ecuador.

${ }^{3}$ Carrera de Ingeniería Industrial, Universidad Politécnica Salesiana, Quito, Ecuador.
}

Forma sugerida de citación: Guilcaso, C.; Ramírez, L.; Coque, A.; Vaca, X.; Molina, D. y Simbaña, I. (2021). «Influencia del recubrimiento de cromato de zinc en la corrosión de los aceros ASTM A-500 y A-500 galvanizado expuestos en una cámara de niebla salina». INGENIUs. N. ${ }^{\circ}$ 26, (julio-diciembre). pp. 63-70. DoI: https://doi.org/10.17163/ings.n26.2020.06. 


\section{Introducción}

El acero ASTM A-500, como tubo rectangular o circular, es comúnmente utilizado para la industria de la construcción, así como en la automotriz, debido a sus propiedades mecánicas y facilidades en la soldadura [1]. Este acero puede ser tratado de diferentes métodos de acuerdo con lo que se requiera, por ejemplo, aumentando la ductilidad al reducir la resistencia. Una aplicación común del acero es para la fabricación de carrocerías de vehículos, automóviles e incluso camiones, de carga y transporte público. El diseño de la carrocería de un vehículo de transporte público se debe realizar considerando el peor escenario ante una colisión y en donde se debe reducir la severidad del accidente para los pasajeros y conductor [2]. Uno de los principales agentes que puede generar fallas de deformación y ruptura es la corrosión, ya que afecta la estructura del acero y, por lo tanto, sus propiedades mecánicas. La aplicación de un recubrimiento anticorrosivo puede prolongar la vida útil de un elemento estructural. Sin embargo, debe aplicarse correctamente, caso contrario, se puede acelerar la corrosión al presentar deslaminaciones o ampollas. La corrosión genera una pérdida de masa, principalmente en la reducción del área de la sección transversal y aunque sea muy baja, puede reducir la resistencia del material y llegar a generar una falla $[3,4]$.

El tratamiento más común utilizado como anticorrosivo es el galvanizado, ya que el Zn provee una protección catódica al acero evitando daños producidos por el óxido, además, es un elemento de bajo costo. Mediante un recubrimiento galvanizado electroquímicamente, un acero mejora su resistencia a la corrosión e incluso sus propiedades mecánicas al modificar ligeramente su composición [5]. El Zn presenta propiedades reciclables y no tóxicas, es por esto por lo que se utilizan más de cinco millones de toneladas por año en aplicaciones anticorrosivas, generando un ahorro de alrededor de USD 2200 millones por reparaciones o reemplazos de elementos oxidados [6].

Para mejorar la resistencia a la corrosión de un acero inmerso en $\mathrm{Zn}$, se pueden añadir diferentes elementos considerando el contenido apropiado. Kania et al. [7] caracterizaron la microestructura y analizaron la resistencia corrosiva de un recubrimiento de $\mathrm{Zn}$ obtenido en una inmersión de Zn-AlNiBi. Los autores utilizaron acero de bajo contenido de Si, 0,021\%, en muestras de $50 \times 100 \times 2 \mathrm{~mm}$ sumergidas por $180 \mathrm{~s}$ en la solución estudiada, a $450{ }^{\circ} \mathrm{C}$. Las muestras se introdujeron en una cámara de niebla salina (CNS) con $\mathrm{NaCl}$ al $5 \%$ a una temperatura de $35 \pm 2{ }^{\circ} \mathrm{C} \mathrm{y}$ $\mathrm{pH}$ entre 6,8 a 7,2 , desde 24 hasta $1000 \mathrm{~h}$. La masa de las muestras fue medida cada $24 \mathrm{~h}$ y los resultados indican que este nuevo recubrimiento es mejor agente anticorrosivo que el Zn puro, ya que la presencia de corrosión se redujo en un $30 \%$, teniendo como masas finales 140,34 y $108,24 \mathrm{~g} / \mathrm{m}^{2}$, respectivamente. $\mathrm{Al}$ inspeccionar el recubrimiento, se aprecia $\mathrm{Bi}$ en la superficie, pero no se visualiza $\mathrm{Al}$ o $\mathrm{Ni}$, por esto, los autores recomiendan utilizar el $\mathrm{Bi}$ como aditivo en lugar del $\mathrm{Pb}$ que es ambientalmente perjudicial.

Previo a considerar un recubrimiento a gran escala para ser aplicado en el sector industrial, deben llevarse a cabo varias pruebas experimentales y la alternativa de mayor viabilidad para analizar la velocidad de corrosión es mediante una CNS. Vera et al. [8] evaluaron recubrimientos anticorrosivos en acero expuesto a un ambiente marino, comparando pruebas de campo con una corrosión acelerada. Se utilizó acero A-36 de $100 \times 100 \times 3 \mathrm{~mm}$ limpiado con chorro y aplicando un recubrimiento alto en Zn y otro de esmalte epóxico. Las pruebas en mar fueron en Chile durante 24 meses y se recolectó $\mathrm{SO}_{2}$ para disolverlo en $\mathrm{Na}_{2} \mathrm{CO}_{3}$ al $5 \%$. La pérdida de espesor estuvo entre 71,9 a $222,2 \mu \mathrm{m}$, dependiendo de las condiciones climáticas, por lo que se estimó una velocidad de corrosión de 131,4 $\mu \mathrm{m} /$ año. Mediante un algoritmo, los autores llegaron a determinar que las condiciones similares en una CNS se deben realizar a $37{ }^{\circ} \mathrm{C}$, con un $100 \%$ de humedad y $\mathrm{NaCl}$ al $3,5 \%$ durante 3000 h de exposición (125 días), reduciendo el tiempo en 6 veces.

La electrodeposición de $\mathrm{Zn}$ nanocristalino en acero para mejorar su resistencia a la corrosión fue analizada por Li et al. [6]. El experimento utilizó solución $\mathrm{NaCl}$ al 3,5 \% y la electrodeposición se realizó con un baño de $\mathrm{ZnSO}_{4}$ con un acero de bajo carbono, después se lavaron las muestras y se secaron. Mediante un microscopio de barrido electrónico (SEM, por sus siglas en inglés), se visualizó que el grano de óxido se redujo de $5 \mu \mathrm{m}$ a $40 \mathrm{~nm}$, por lo que la aplicación de este recubrimiento aumentó casi en 40 veces la resistencia a la corrosión.

Stojanović et al. [9] evaluaron la protección a la corrosión de un sistema de dos recubrimientos en un ambiente marino simulado. Placas de acero naval de $120 \times 70 \times 3 \mathrm{~mm}$ fueron limpiadas con chorro para aplicar dos capas de recubrimiento anticorrosivo de $150 \mu \mathrm{m}$ cada una y posteriormente un recubrimiento antiincrustante. Las placas fueron expuestas durante $1440 \mathrm{~h}$ en una CNS al 38,08 \% y un pH de 8, donde se seleccionó dos grupos, unos para inmersión y otro para agitación. Los resultados indican que las placas que fueron agitadas en la solución generan mayor microorganismos y como resultado, una mayor corrosión, además que el segundo recubrimiento no tuvo la adherencia adecuada, por lo que los autores recomiendan analizar la composición química de los recubrimientos anticorrosivos.

El comportamiento corrosivo del acero ASTMSA213-T22 recubierto con $\mathrm{Cr}_{2} \mathrm{O}_{3}$ en un ambiente salino a $700{ }^{\circ} \mathrm{C}$ fue analizado por Goyal et al. [10]. Las muestras fueron de $22 \times 15 \times 3 \mathrm{~mm}$ y recubiertas con $\mathrm{Cr}_{2} \mathrm{O}_{3}$ comercial con un espesor entre 250 a $255 \mu \mathrm{m}$. 
Se utilizó un horno para generar corrosión en caliente con $\mathrm{Na}_{2} \mathrm{SO}_{4}$ al $60 \%$ durante $1 \mathrm{~h}$ y se consideró la masa final de las muestras. El material base presentó una mayor velocidad de corrosión comparado con el recubrimiento de $\mathrm{Cr}_{2} \mathrm{O}_{3}$ que presentó una perfecta adherencia, generando que la masa del material permaneciera en un $94,5 \%$.

Esta investigación está dirigida a determinar la influencia de un recubrimiento de cromato de zinc en la corrosión de los aceros ASTM A-500 y A-500 galvanizado, acero utilizado en la fabricación de carrocerías de vehículos de transporte público. De esta manera, se va a establecer si se requiere este tratamiento previo. La sección de Materiales y métodos describe los pasos del proceso experimental considerando diferentes normas para su realización. En Resultados se presentan figuras y el análisis comparativo de las muestras después de la exposición a la CNS. Finalmente, las Conclusiones explican los resultados obtenidos y definen cuál acero tuvo menor corrosión.

\section{Materiales y métodos}

La Figura 1 resume el procedimiento considerado para el desarrollo experimental y obtención de resultados de esta investigación.

\subsection{Preparación del material}

El material utilizado fue un tubo de acero al carbono ASTM A-500 obtenido mediante soldadura en frío y sin cordón, utilizado para la fabricación de carrocerías. Mediante una matriz de diseño experimental, se estableció que se requieren ocho probetas de cada material, para ello, fueron cortadas mediante disco abrasivo utilizando una amoladora y así conseguir placas de $100 \times 50 \times 2 \mathrm{~mm}$.

\subsection{Limpieza superficial}

La Sociedad de Recubrimientos Protectores, (SSPC, por sus siglas en inglés) ha normado ciertos procedimientos de limpieza superficial requeridos antes de la aplicación de un recubrimiento anticorrosivo. Para esta investigación, se ha optado por dos métodos de limpieza superficial, el primero consiste en una limpieza mecánica, de acuerdo con la norma SSPC-SP-3 [11], utilizando lijas para remover capas de óxido. Posteriormente, se requiere de un cepillo de alambre rotativo como herramienta eléctrica para limpiar la superficie y remover todos los restos de escombros. Es importante mencionar que, debido a la geometría tanto del material, así como de las herramientas, es probable que puedan quedar desechos ocultos en las irregularidades del material.

Para el segundo método de limpieza superficial, se consideró la norma SSPC-SP-5 [12] que consiste en la aplicación de un líquido presurizado para remover todas las partículas sobre la superficie del material. Este método asegura una limpieza total, libre de deslaminaciones, ya que un chorro presurizado permite retirar incluso polvo y grasa, y así lograr una mejor adherencia del recubrimiento.

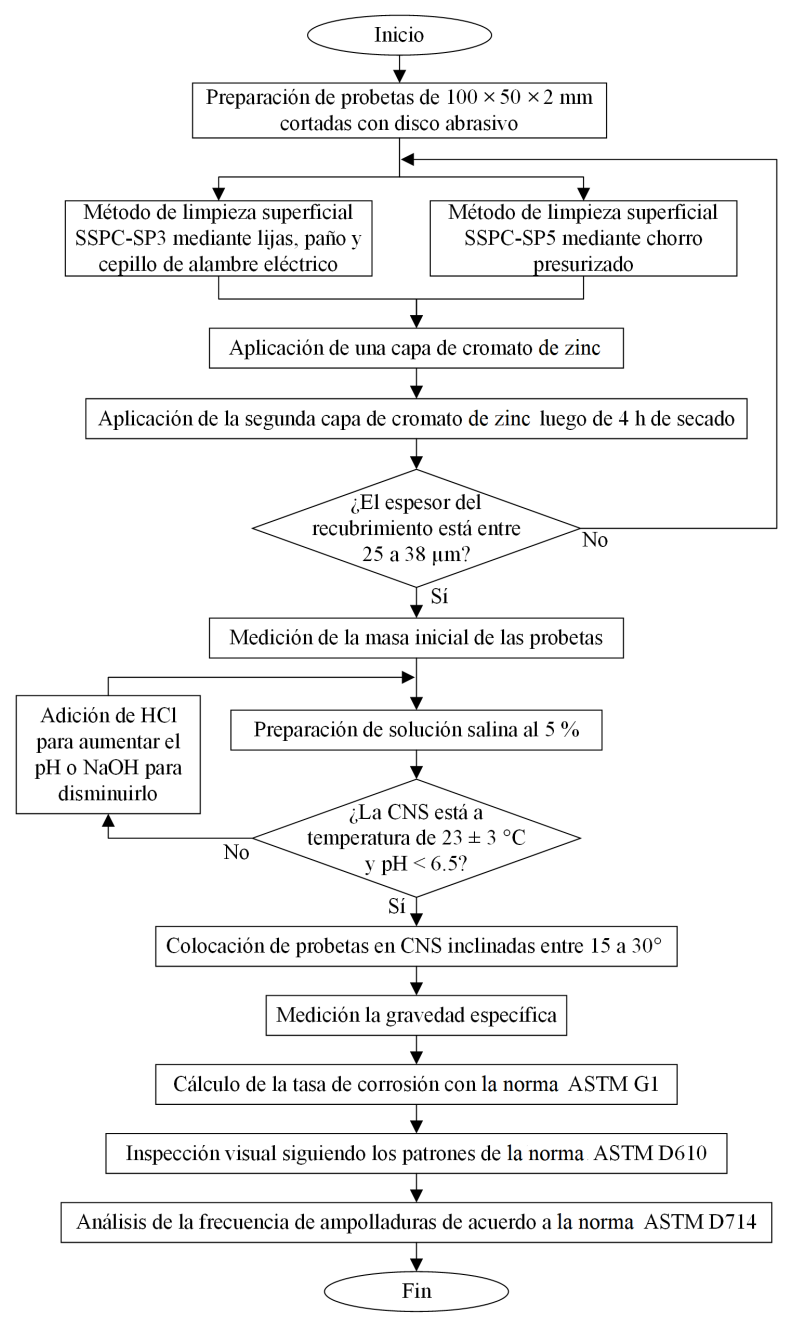

Figura 1. Diagrama de flujo para el desarrollo experimental

\subsection{Recubrimiento}

Un recubrimiento de cromato de zinc se aplicó sobre las ocho probetas de A-500 y acero galvanizado, cuatro a ser limpiadas mediante SSCP-SP-3 y las otras cuatro con SSCP-SP-5. Este recubrimiento consta de resinas y pigmentos que proveen resistencia corrosiva ante los diferentes agentes atmosféricos, además, es uno de los más utilizados comercialmente.

El recubrimiento fue diluido en thinner en una proporción de 4 a 1 para su aplicación mediante soplete. El espesor del recubrimiento según el fabricante debe estar entre 25 a $38 \mu \mathrm{m}$, por lo cual se aplicaron dos capas, con un tiempo de secado de $4 \mathrm{~h}$ a $20{ }^{\circ} \mathrm{C}$ 
entre ellas. Para realizar la medición del espesor del recubrimiento, se utilizó un medidor de espesor de revestimientos Elecometer 456.

\subsection{Ensayo en la CNS}

La norma ASTM B-117 [13] se utilizó para la experimentación en la CNS, donde se indica la preparación, procedimientos, predicción y resultados bajo un ambiente corrosivo controlado. Se utilizó solución salina al $5 \%$, con $12,72 \mathrm{~kg}$ de $\mathrm{NaCl}$ en un volumen de 240 litros de agua destilada.

Entre los instrumentos de medida que se utilizaron, es importante mencionar el medidor de $\mathrm{pH}$ a prueba de agua HANNA HI9125, para verificar que el $\mathrm{pH}$ fuera menor a 6,5 a una temperatura promedio de $23{ }^{\circ} \mathrm{C}$. $\mathrm{Si}$ el valor de $\mathrm{pH}$ baja, se puede añadir $\mathrm{NaOH}$, caso contrario, cuando el $\mathrm{pH}$ es alto, se añade $\mathrm{HCl}$ hasta obtener el valor requerido. Mediante un medidor de flujo, se recolectó $2 \mathrm{ml} / \mathrm{h}$ de $\mathrm{NaCl}$ de la CNS cada 24 horas. Un refractómetro digital MA887 como medidor de salinidad se utilizó para determinar la gravedad específica del $\mathrm{NaCl}$. La masa de las probetas, de material base y con el recubrimiento, se midió previo a ingresarlas a la CNS, donde fueron colocadas con una inclinación de 20 a $30^{\circ}$.

Para el recubrimiento de cromato de zinc, el tiempo promedio para este ensayo se estima en $250 \mathrm{~h}$. Se establecieron diferentes tiempos de exposición dentro de la CNS, teniendo 200, 250 y $350 \mathrm{~h}$ para determinar cómo influye este tiempo de exposición en la corrosión del acero. Después de cada período, se tomó la medida del peso de las probetas nuevamente, así calcular la pérdida de masa entre el valor inicial y el final.

\subsection{Velocidad de corrosión}

Según la norma ASTM G-1 [14], este parámetro se define como la pérdida de espesor de un acero por unidad de tiempo para analizar el daño ocasionado por corrosión después de la exposición. La velocidad de corrosión $(\dot{C})$ depende del material, así como del tiempo de exposición, y puede ser obtenida mediante:

$$
\dot{C}=(K \cdot W) \cdot(A \cdot t \cdot \delta)^{-1}
$$

Donde $K$ permanece constante con un valor de $8,76 \times 10^{4} \mathrm{~mm} /$ año, $W$ es la pérdida de masa expresada en $\mathrm{g}, A$ es el área de la probeta en $\mathrm{cm}^{2}, t$ son las horas de exposición y $\delta$ es la densidad del acero en $\mathrm{g} / \mathrm{cm}^{3}$.

\subsection{Evaluación visual}

Para evaluar el grado de corrosión sobre una superficie recubierta, se consideró la norma ASTM D-610 [15] para determinar si el recubrimiento debía ser reparado o reemplazado. Según esta norma, se ha establecido una escala desde 1 a 10 en función del área superficial de corrosión, donde 10 se indica para manchas de óxido que son $0,01 \%$ menores al área total, mientras que si el área corroída es mayor al $33 \%$, se designa 1. Este número debe ir acompañado de una letra para indicar el patrón visual de corrosión, manchado $(S)$, puntual $(P)$ o general $(G)$.

Esta evaluación visual es consolidada con una segunda inspección, que consiste en evaluar las ampollas producidas por corrosión sobre el recubrimiento según la norma ASTM D-714 [16]. Para la identificación de ampollas, se ha asignado un número entre 0 a 10 , donde 10 es una superficie sin imperfecciones, 8 indica pequeñas ampollas difíciles de apreciar a simple vista y los números inferiores indican ampollas cada vez más grandes. Además, este número va acompañado de una letra que representa la frecuencia de presencia de ampollas por unidad de área, indicando si esta frecuencia es poca $(F)$, media $(M)$, media densa $(M D)$ o densa $(D)$.

\section{Resultados y discusión}

\subsection{Velocidad de corrosión}

Al considerar la diferencia entre la masa inicial y final de las probetas, se pudo calcular la corrosión por año. A continuación, se presentan los valores de velocidad de corrosión obtenidos mediante la Ecuación (1) para el material base y las probetas con los métodos de limpieza superficial SSPC-SP-3 y SSPC-SP-5.

Las probetas de acero ASTM A-500 y el acero galvanizado sin el recubrimiento de cromato de zinc presentaron una velocidad de corrosión de 1,672 y 0,535 mm/año luego de $200 \mathrm{~h}$ de exposición en la CNS, como se muestra en la Figura 2a. Mientras que con la limpieza mecánica SSPC-SP-3, los valores fueron de 0,129 y 0,044 mm/año para el A-500 y el acero galvanizado, respectivamente, con el mismo tiempo de exposición. Finalmente, para las probetas de A-500 y acero galvanizado que fueron limpiadas a chorro según la norma SSPC-SP-5, se tuvo una velocidad de corrosión de 0,051 y 0,034 mm/año. Los valores de velocidad de corrosión tienden a incrementarse a medida que el tiempo de exposición aumenta, sin embargo, el óxido sobre la superficie de las probetas puede crear un recubrimiento adicional y este valor puede disminuir.

Después de $250 \mathrm{~h}$ de exposición, las probetas de A-500 con los métodos de limpieza superficial y recubrimiento de cromato de zinc disminuyeron el valor de la velocidad de corrosión, teniendo 0,083 y 0,014 mm/año para los métodos SP-3 y SP-5, respectivamente, mientras que el material base aumentó su corrosión a 2,092 mm/año. Con el mismo tiempo de exposición, el acero galvanizado presentó resultados opuestos, donde el material base redujo su corrosión a 0,436 mm/año y los métodos SP-3 y SP-5 se incre- 
mentaron en 0,058 y 0,002 mm/año, respectivamente, con respecto a la exposición de $200 \mathrm{~h}$.

Finalmente, con un tiempo de exposición de $350 \mathrm{~h}$, las probetas de material base de A-500 y acero galvanizado tuvieron una corrosión de 1,594 y 0,142 mm/año. Para la limpieza superficial SSPC-SP-3, estos aceros presentaron una velocidad de corrosión de 0,139 y 0,103 mm/año, respectivamente. Además, por el método de limpieza superficial SP-5, las probetas registraron valores de 0,033 y 0,029 mm/año para el A-500 y el acero galvanizado, respectivamente.

Los valores obtenidos están más agrupados con la limpieza mediante SSPC-SP-5, teniendo una desviación estándar de 0,0185 y 0,0036 mm/año para el A-500 y el acero galvanizado, respectivamente. Estos aceros presentaron una desviación estándar con el método SSPCSP-3 de 0,0299 y 0,03378 mm/año, respectivamente, además, 0,2679 y 0,2044 mm/año sin tratamiento.

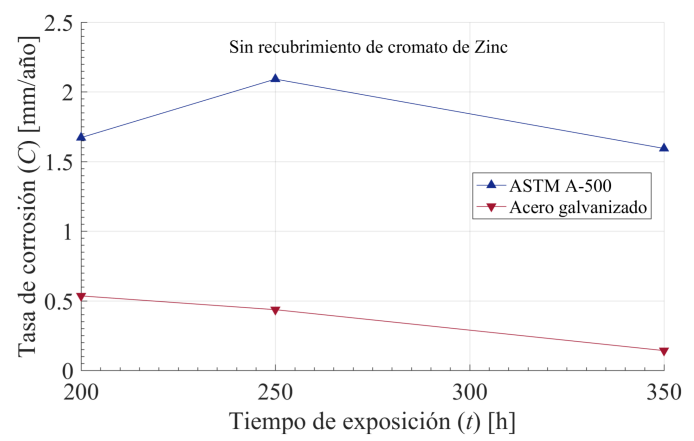

(a)

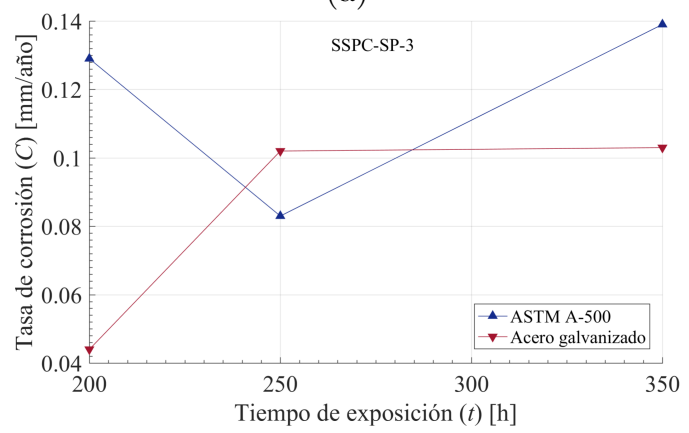

(b)

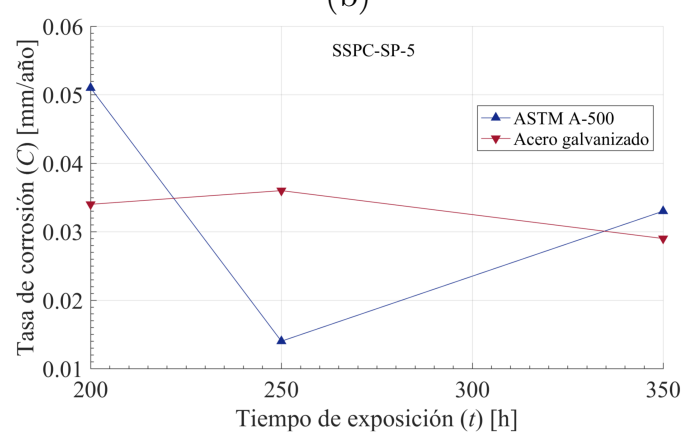

(c)

Figura 2. Velocidad de corrosión para probetas a) sin recubrimiento, b) limpieza SSPC-SP-3, c) limpieza SSPCSP-5

\subsection{Evaluación visual}

Mediante un microscopio SEM, se visualizó la morfología de los productos de corrosión en las probetas de A-500. En la Figura 3a, se muestra una probeta sin recubrimiento expuesta a $300 \mathrm{~h}$, observando una formación generada por la corrosión que se conoce como lepidocrocita. La Figura 3b presenta el análisis de morfología de la probeta con un tiempo de exposición de 350 h, donde se identifica goetita semicristalina en forma de nubes, así como láminas muy delgadas de lepidocrita, con un contorno similar a raíces.

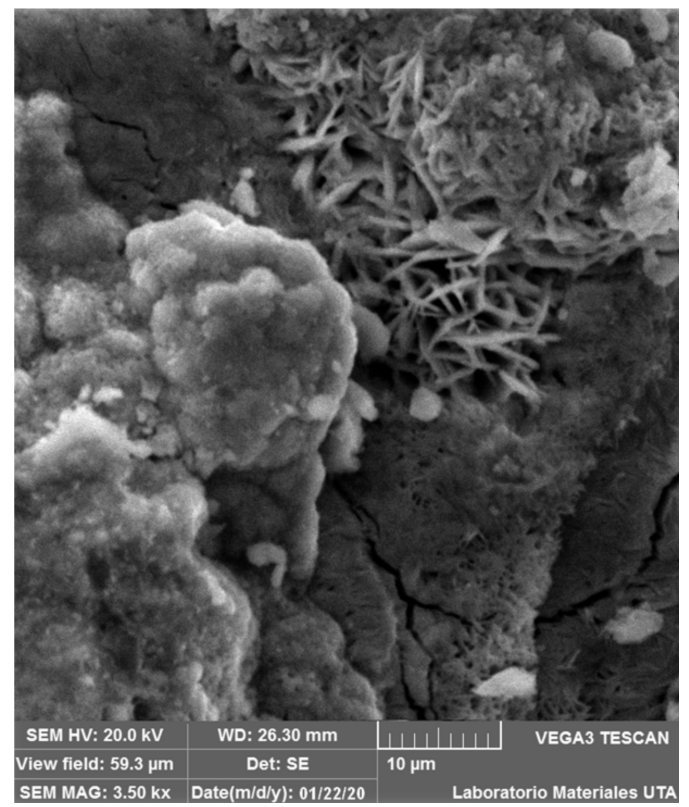

(a)

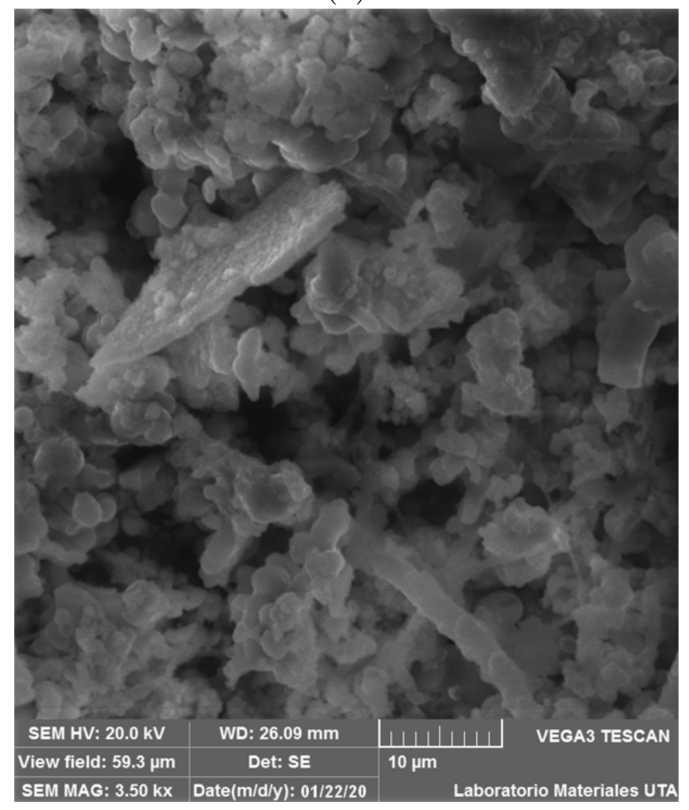

(b)

Figura 3. Microestructura del acero ASTM A-500 sin recubrimiento expuesto en la CNS a) $300 \mathrm{~h}$, b) $350 \mathrm{~h}$ 
La Figura 4a indica los resultados de la corrosión existente en el ASTM A-500, mientras que en la Figura 4b se aprecia el acero galvanizado corroído, luego de 200, 250 y 350 h de exposición en la CNS. Los aspectos que presentan las probetas son de superficies con mucha corrosión, visible a simple vista, e incluso con cúmulos significativos, corroborando los valores calculados en la velocidad de corrosión.

Las probetas de ASTM A-500, con previa limpieza superficial SSPC-SP-5 a la aplicación del cromato de zinc, se indican en la Figura 5b, luego de tiempos de exposición de 200, 250 y 350 h, respectivamente. Para la probeta que estuvo expuesta por $200 \mathrm{~h}$, se ha asignado una corrosión de $4 \mathrm{G}$, mientras que las probetas con tiempo de exposición de 250 y 350 h, fueron evaluadas como 5 G. En ninguna de estas probetas se pudo evidenciar la existencia de ampollas, teniendo una buena adherencia del recubrimiento.
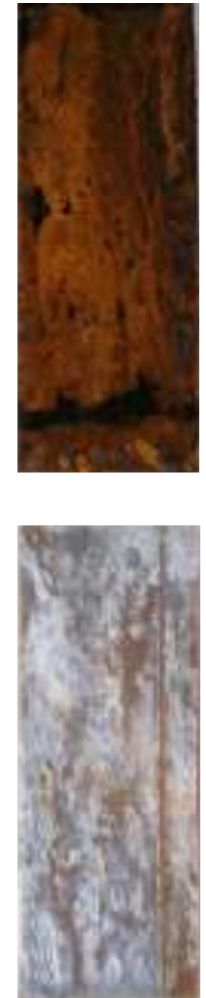

$200 \mathrm{~h}$

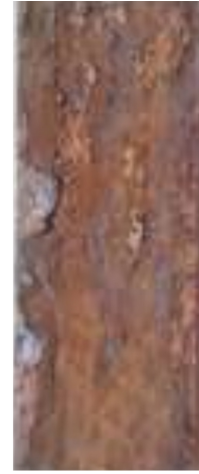

(a)

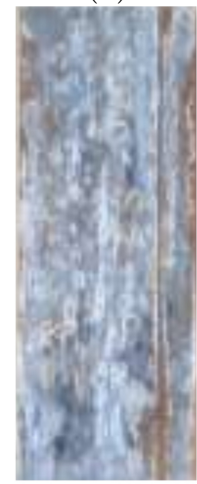

$250 \mathrm{~h}$

(b)

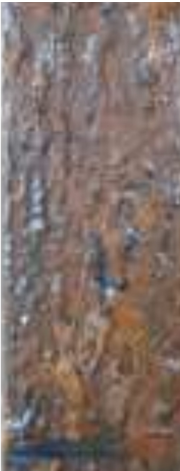

$200 \mathrm{~h}$

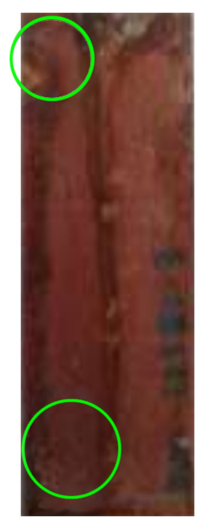

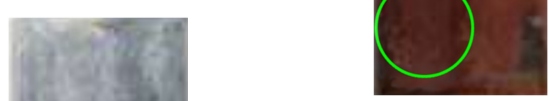

a) ASTM A-500, b) acero galvanizado

Los patrones de la norma ASTM D-610 se utilizaron como referencia para realizar una evaluación del porcentaje de superficie corroída. Además, esta evaluación visual se complementó indicando la frecuencia y tamaño de ampollas presentes en las probetas según la norma D-714. En la Figura 5a se muestran las probetas de A-500 con limpieza superficial SSPC-SP-3 recubiertas, donde se han señalado manchas de corrosión en un $3 \%$, teniendo un $5 \mathrm{~S}$ luego de $200 \mathrm{~h}$. Para la probeta expuesta por $250 \mathrm{~h}$, se ha considerado una corrosión de $5 \mathrm{G}$ y se ha evaluado como $3 \mathrm{G}$ a la probeta que estuvo en la CNS por $250 \mathrm{~h}$. La frecuencia de las ampollas fue medida para las probetas con tiempo de exposición de 200 y 350 h, indicando una evaluación de $6 \mathrm{M}$ y $2 \mathrm{M}$, respectivamente. Mientras que en la probeta de $250 \mathrm{~h}$ de exposición esta frecuencia fue mayor, asignándole un valor de $6 \mathrm{MD}$ con ampollas visibles.
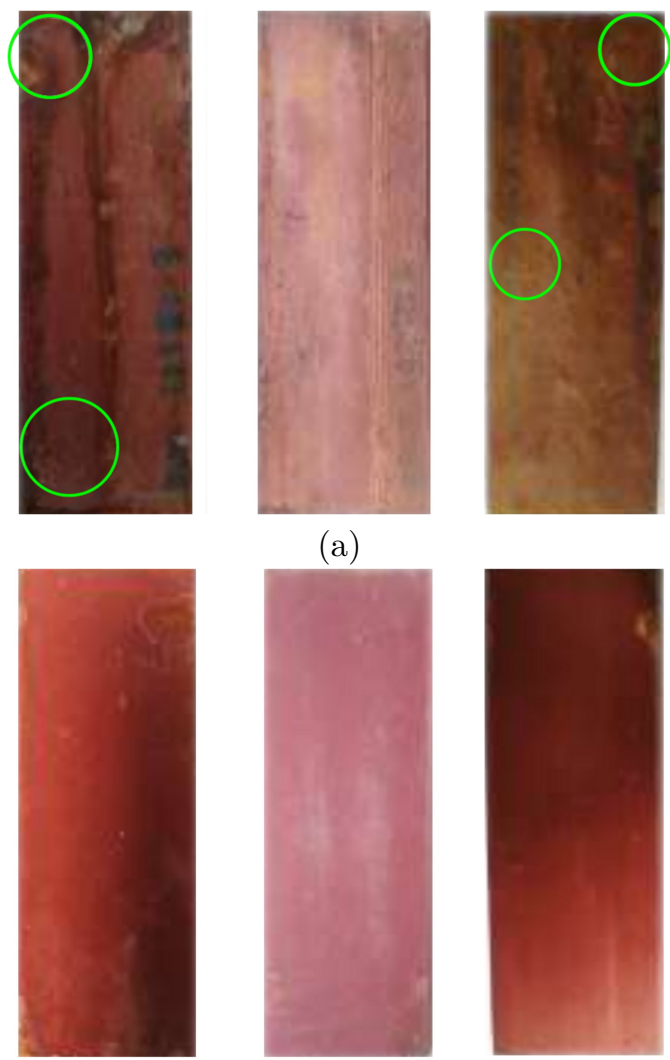

(a)

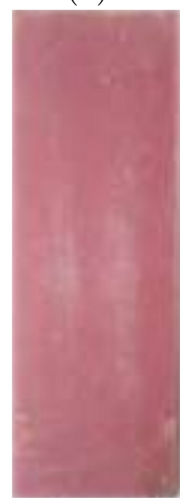

$250 \mathrm{~h}$

(b)

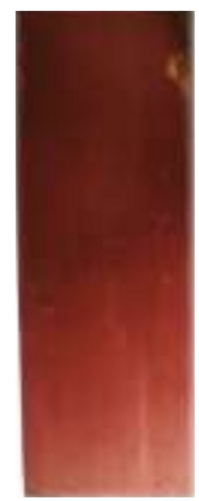

$350 \mathrm{~h}$

Figura 5. Probetas de ASTM A-500 expuestas a 200, 250 y 350 h, a) SSPC-SP-3, b) SSPC-SP-5

La Figura 6a presenta las probetas de acero galvanizado limpiadas con el método SSPC-SP-3 y recubiertas con cromato de zinc. En la probeta que estuvo en la CNS por $200 \mathrm{~h}$, se han evidenciado manchas de corrosión, teniendo $5 \mathrm{~S}$ y no se registraron ampollas sobre su superficie. Para la probeta expuesta por $250 \mathrm{~h}$, la corrosión visible es general, teniendo $6 \mathrm{G}$ y se visualizaron pequeñas ampollas, por lo que se le ha asignado $6 \mathrm{M}$. A la probeta con tiempo de exposición de $350 \mathrm{~h}$, se le ha asignado una corrosión de $4 \mathrm{~S}$ por manchas visibles y la frecuencia de ampollas fue poca, aunque considerables, por lo que se tiene $6 \mathrm{~F}$. Las probetas de acero galvanizado con limpieza SSPC-SP-5 se muestran en la Figura 6b. 

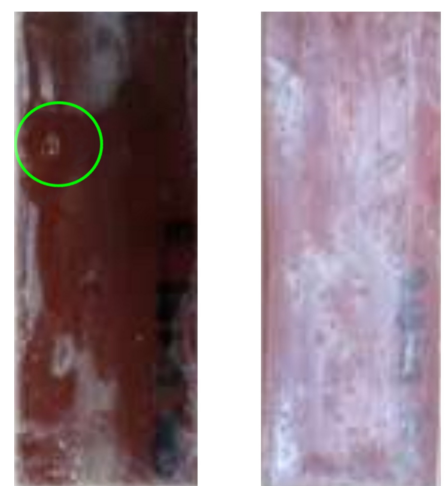

(a)

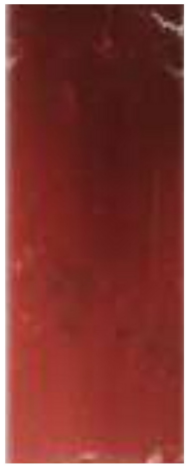

$200 \mathrm{~h}$

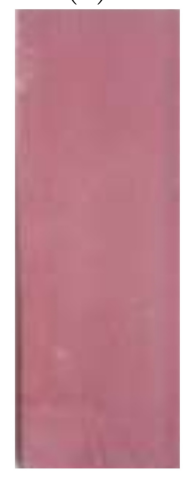

$250 \mathrm{~h}$

(b)
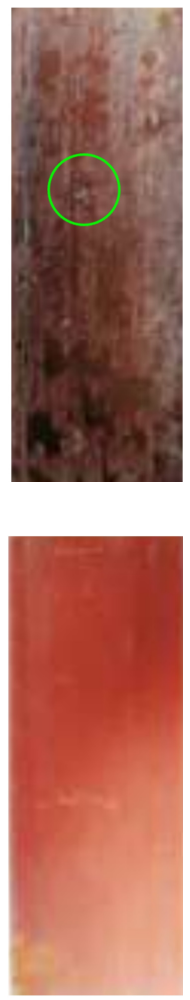

$350 \mathrm{~h}$
Figura 6. Probetas de ASTM A-500 galvanizado expuestas a 200, 250 y 350 h, a) SSPC-SP-3, b) SSPC-SP-5

En la probeta de acero galvanizado con tiempo de exposición de $250 \mathrm{~h}$ no se ha podido visualizar señales de corrosión y existen pequeñas ampollas, teniendo una frecuencia de $8 \mathrm{M}$. Mientras que las probetas expuestas por 200 y $350 \mathrm{~h}$, muestran pequeñas manchas de corrosión, por lo que se les ha asignado $5 \mathrm{~S}$ y ninguna de ellas ha presentado ampollas sobre sus superficies.

\section{Conclusiones}

Los valores obtenidos de velocidad de corrosión de las probetas indican que la corrosión afecta más durante las primeras horas de exposición. Para el acero A-500 luego 200 y 350 h de exposición, se tuvo una velocidad de corrosión de 1,672 y 1,594 mm/año, respectivamente. Para el acero galvanizado sin recubrimiento, en los mismos tiempos se tuvo valores de 0,535 y $0,142 \mathrm{~mm} /$ año. Esta tendencia se mantuvo durante los ensayos de las probetas limpiadas mediante SSPC-SP3 y SSPC-SP5 de los dos materiales.

El acero A-500 sin tratamiento tuvo una máxima velocidad de corrosión de 2,092 mm/año, en la probeta expuesta por $250 \mathrm{~h}$ a la CNS, siendo 3,9 veces el valor más alto que el alcanzado por el acero galvanizado. Con el método SSPC-SP-3, el acero A-500 y el acero galvanizado alcanzaron un máximo de 0,139 y 0,103 mm/año, teniendo una diferencia del 25,9\%. Con la limpieza según la norma SSPC-SP-5, la velocidad de corrosión más alta fue de 0,051 y 0,036 mm/año para el A-500 y el acero galvanizado, respetivamente. Entonces, en los aceros sin recubrimiento es evidente que el galvanizado es una protección anticorrosiva, aunque continúa presentando valores más elevados que con recubrimiento. La corrosión puede considerarse similar en los dos materiales, pero el método SSPC-SP-5 brinda menor corrosión en las probetas.

$\mathrm{Al}$ considerar el análisis entre los dos métodos de limpieza superficial, según la norma ASTM D-610, se tuvo que, el A-500 y el acero galvanizado limpiados con el método SSPC-SP-3 presentaron una corrosión superficial general y manchado entre $5 \mathrm{~S}$ y $5 \mathrm{G}$ a $6 \mathrm{G}$. Mientras que esta evaluación se redujo en las probetas tratadas con la limpieza SSPC-SP-5, considerando a las probetas con una corrosión general $4 \mathrm{G}$.

La norma ASTM D-714 se utilizó para complementar la evaluación visual y determinar la frecuencia y tamaño de las ampollas. En las probetas limpiadas según la norma SSPC-SP-3 de A-500 y acero galvanizado, se llegó a tener una evaluación máxima y mínima de $2 \mathrm{M}, 6 \mathrm{MD}$ y $6 \mathrm{M}, 6 \mathrm{~S}$, respectivamente. Por este motivo se puede afirmar que, el recubrimiento de cromato de zinc se adhiere de manera similar en los dos materiales y brinda una protección anticorrosiva 7,7 y 8,2 veces superior que el A-500 y el acero galvanizado sin recubrimiento, respectivamente.

\section{Referencias}

[1] M. Morrison, "A ductile fuse for special concentrically braced frames," in Ninth International Conference on Advances in Steel Structures (ICASS2018)At: Hong Kong, 2018. [Online]. Available: https://bit.ly/3xJJqcW

[2] K. B. Yu., G. Stradanchenko S., S. A. Yu., S. Garmider A., and B. Kalmykova Yu., "Effect of the bus bodywork on impact strength properties in roll-over," ARPN Journal of Engineering and Applied Sciences, vol. 11, no. 17, pp. 10205-10208, 2016. [Online]. Available: https://bit.ly/3vsZf5F

[3] C. Cui, A. T. O. Lim, and J. Huang, "A cautionary note on graphene anti-corrosion coatings," Nature Nanotechnology, vol. 12, no. 9, pp. 834-835, Sep. 2017. [Online]. Available: https://doi.org/10.1038/nnano.2017.187

[4] K. K. Toledo, H.-S. Kim, Y.-S. Jeong, and I.-T. Kim, "Residual compressive strength of short tubular steel columns with artificially fabricated local corrosion damage," Materials, vol. 13, no. 4, 2020. [Online]. Available: https://doi.org/10.3390/ma13040813 
[5] V. N. Tseluikin and A. A. Koreshkova, "Corrosion resistance of composite coatings based on zinc," Chemical and Petroleum Engineering, vol. 52, no. 7 , pp. 560-562, Nov. 2016. [Online]. Available: https://doi.org/10.1007/s10556-016-0232-3

[6] Q. Li, H. Lu, J. Cui, M. An, and D. Li, "Electrodeposition of nanocrystalline zinc on steel for enhanced resistance to corrosive wear," Surface and Coatings Technology, vol. 304, pp. 567-573, 2016. [Online]. Available: https://doi.org/10.1016/j.surfcoat.2016.07.056

[7] H. Kania, M. Saternus, J. Kudláçek, and J. Svoboda, "Microstructure characterization and corrosion resistance of zinc coating obtained in a zn-alnibi galvanizing bath," Coatings, vol. 10, no. 8, 2020. [Online]. Available: https://doi.org/10.3390/coatings10080758

[8] R. Vera, E. Cruz, M. Bagnara, R. Araya, R. Henríquez, A. Díaz-Gómez, and P. Rojas, "Evaluation of anticorrosive coatings on carbon steel in marine environments: Accelerated corrosion test and field exposure," International Journal of Electrochemical Science, vol. 13, pp. 898-914, 2018. [Online]. Available: https://doi.org/10.20964/2018.01.66

[9] I. Stojanović, A. Farkas, V. Alar, and N. Degiuli, "Evaluation of the corrosion protection of two underwater coating systems in a simulated marine environment," $A d$ vances in Surface Engineering, vol. 71, no. 12, pp. 4330-4338, Dec. 2019. [Online]. Available: https://doi.org/10.1007/s11837-019-03669-4

[10] K. Goyal, H. Singh, and R. Bhatia, "Hot-corrosion behavior of Cr2O3-CNT-coated ASTM-SA213-
T22 steel in a molten salt environment at $700^{\text {circ }} \mathrm{C}$," International Journal of Minerals, Metallurgy, and Materials, vol. 26, no. 3, pp. 337-344, Mar. 2019. [Online]. Available: https://doi.org/10.1007/s12613-019-1742-8

[11] SSPC, "Sspc-sp-3, surface preparation specification: Power tool cleaining," The Society for Protective Coatings, Tech. Rep., 2003. [Online]. Available: https://bit.ly/3u4JX6Z

[12] _ , "Sspc-sp-5, white metal blast cleaning," The Society for Protective Coatings, Tech. Rep., 1999. [Online]. Available: https://bit.ly/3e3L2q1

[13] ASTM, "Astm b117 - 19 standard practice for operating salt spray (fog) apparatus," ASTM International, West Conshohocken, PA, Tech. Rep., 2019. [Online]. Available: http://doi.org/10.1520/B0117-19

[14] — , "Astm g1 - 03(2017)e1 standard practice for preparing, cleaning, and evaluating corrosion test specimens," ASTM International, West Conshohocken, PA, Tech. Rep., 2017. [Online]. Available: http://doi.org/10.1520/G0001-03R17E01

[15] — - "Astm d610 - 08(2019) standard practice for evaluating degree of rusting on painted steel surfaces," ASTM International, West Conshohocken, PA, Tech. Rep., 2019. [Online]. Available: http://doi.org/10.1520/D0610-08R19

[16] — - "Astm d714 - 02(2017) standard test method for evaluating degree of blistering of paints," ASTM International, West Conshohocken, PA, Tech. Rep., 2017. [Online]. Available: http://doi.org/10.1520/D0714-02R17 